\title{
A Novel High Resolution E-Field Microscope System with Applications in HPA Diagnostics
}

\author{
N. Dehghan ${ }^{1}$, A. Porch ${ }^{1}$, S.C. Cripps ${ }^{1}$, and P. H. Aaen ${ }^{2}$ \\ Cardiff University, UK \\ (Freescale Semiconductor, Tempe,U.K)
}

\begin{abstract}
A new type of high resolution E-field microscope system is described. The system is based on a novel design of an active E-field probe (EFP) probe, which is both nonintrusive and displays high spatial resolution. This paper focuses on the construction and spatial resolution of the probe, which shows significant evolution from that previously reported [1], and some measurements on passive structures which indicate that features smaller than 100 microns can be individually resolved. The probe construction lends itself to further improvements in spatial resolution. Measurement results are presented which demonstrate the high resolution of the probe, and its potential utility in measuring waveforms with high spatial resolution at individual points on microwave circuits, discrete devices, and integrated circuits.
\end{abstract}

\section{INTRODUCTION}

$\mathrm{W}$ ITH the development of ever increasingly sophisticated high frequency systems, it vital to have an understanding each component and their interactions. The aid of electromagnetic simulation software has allowed designers to reduce the number of prototype systems produced, as errors and design flaws can usually be flagged before fabrication. This does method does not pinpoint all potential errors. The development of a non-intrusive E-field microscope system has proved to be vital as a method of evaluating the field distribution at a device plane, as each component may not perform in the expected manner in real life application.

This paper describes the design and fabrication of an E-field probe, and its in-situ applications. The design of the EFP, is a development of a probe described in [1], and is constructed using an open-ended co-axial transmission line which can respond to local normal electric fields with a spatial resolution of about 100 microns.

From previous papers [1], knowing that the probed signal is small and the unwanted presence of stray-up problematic, the design of the EFP includes an amplifier close to the probe tip. This feature evidently amplifies the small signal but also eliminate the stray pickup form the feeder cable.

\section{Probe CONSTRUCtion}

The basis of the EFP is an open-ended co-axial transmission line, which can be shown to have an increasingly sharp radiation pattern as the inner conductor is retracted inside the outer sleeve [2]. The use of such a retracted configuration is an important novel feature of the probe design, and differentiates it from previously published work in this area [2].

Conventional EM thinking, backed up by simulations [3], suggests that the spatial resolution of such a probe will be limited to the diameter of the inner conductor. On this basis, the spatial resolution can be improved indefinitely by using thinner co-ax. But a practical limitation becomes evident; as the capture area of the probe is reduced, the amount of coupling also reduces, and the desired signal soon becomes indistinguishable with the stray pickup on the co-axial leads. In previous literature [4], the EFP is coupled to a resonant transmission line in order to counteract this problem in order to achieve a high spatial resolution with thinner inner conductor.

This problem is exacerbated by the high reflection presented by the probe to the $50 \mathrm{Ohm}$ co-axial measurement system. Both of these problems can be greatly alleviated by the use of a buffer amplifier located close to the probe tip, and this is the second novel feature we describe in this paper .

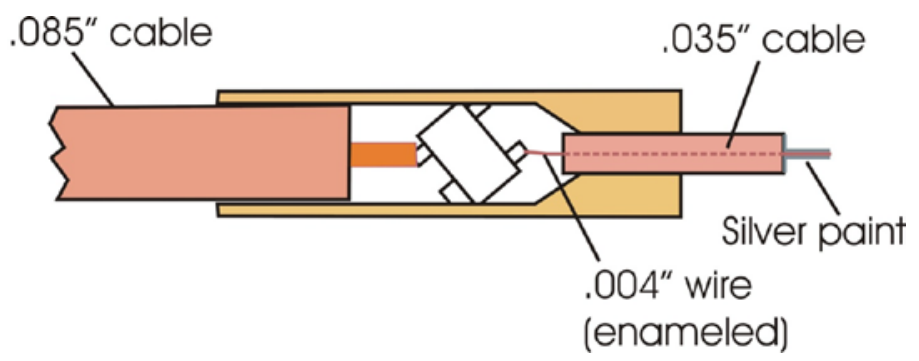

Fig. 1. Schematic diagram of E-field probe.

Such a configuration requires some innovative construction techniques, which have been substantially improved since the basic design was initially reported [1]. The construction of the present probe is shown in Figure 1. A small brass ferrule is machined to take a small diameter semi-rigid cable $(1 \mathrm{~mm}$ dia. approx.) at one end, and a conventional .085 semi-rigid feed cable at the other. Inside the ferrule there is just sufficient space to emplace an SOT packaged, small signal PHEMT transistor (Avago ATF34143). The ferrule has a tight fitting 
cover (shown removed in Figure 2). Although useful results can be obtained by simply flush-cutting the end of the protruding $1 \mathrm{~mm}$ cable, much higher resolution can be obtained by removing the original inner conductor and replacing it with a much thinner piece of enameled copper wire. The actual probe then comprises the short protrusion of the wire beyond the semi-rigid cable, and this is transformed into a very thin co-axial line by coating it with conductive silver paint. The wire diameter for the present set of reported measurements was 100 microns (.004 inch).



Fig. 2. Picture of the ferrule with the cover removed.

\section{MeAsurements.}

Figure 3 shows a 3-D view of a typical test structure that is being used to demonstrate the probe spatial resolution. It consists of a microstrip line which has an end termination consisting of a fan of multiple 20 micron ground bonds. Various structures were measured, having different bondwire counts and different spacings. Figure 4 shows the measured spatial response as the probe is moved transversely along the bond wires shown in Figure 3.

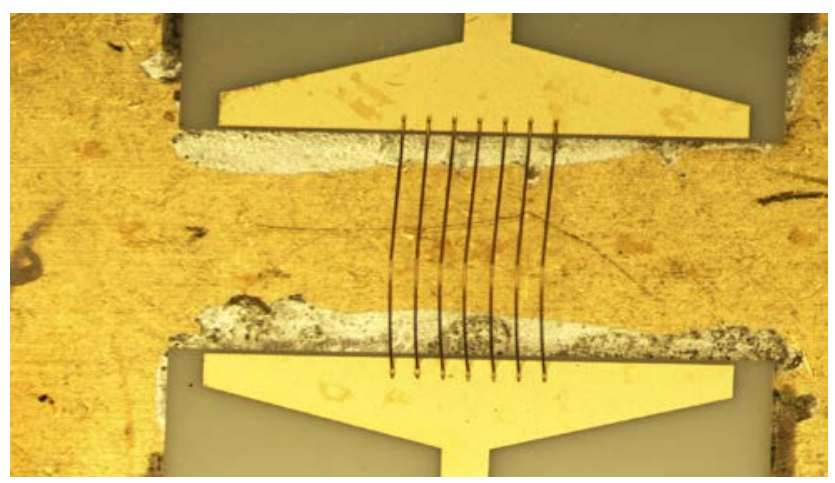



Fig.3. Plan and side view of a 9 bond test structure.
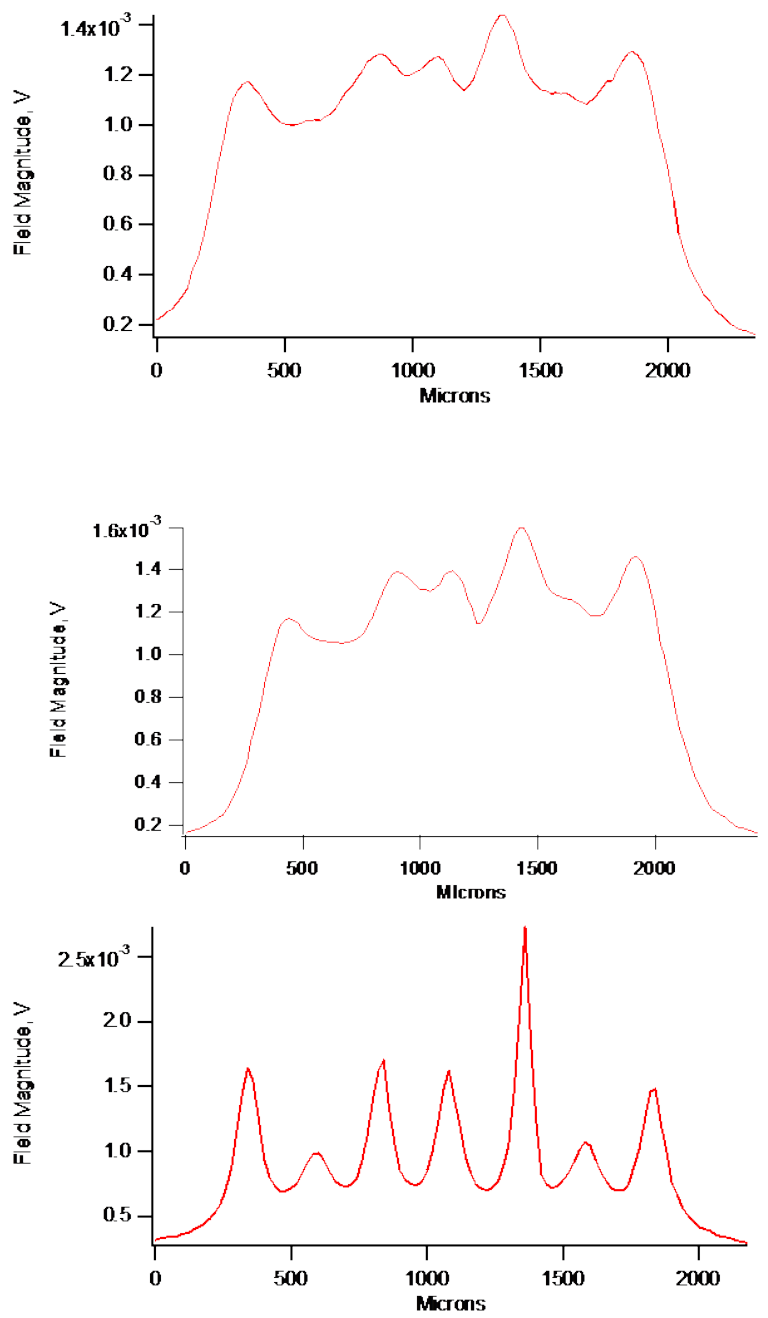

Fig. 4. Voltage distribution transversely along the 9 bond structure shown in Fig 3 Shown in the order, Probe 1, Probe 2 and Probe 3. Probe inner conductor diameters: Probe $1: 0.007$ inch. Probe 2: 0.005 inch. Probe 3:0.004 inch (wire probe)

In previous work [1] we have presented evidence that this probe geometry responds only to the electric field, and almost entirely to the vertical component of the local electric field at the measurement tip; this in turn bears a close physical relationship with the voltage on the wires. Figure 4 shows the 
same measurement using three different probe inner diameters. It is interesting to note that the thinnest available semi-rigid cable has an inner conductor diameter of .005”, which is only slightly larger than the wire probe, yet the wire probe clearly has substantially higher spatial resolution.

Figure 5 shows a measurement taken along a more extensive bondwire structure, having 19 bondwires and closer spacing. Figure 6 shows the 3-D view of the 19 bondwire structure. It is clear that the magnitude of the field peaks are very sensitive to the planarity of the bondwires, but there is a clear overall trend visible, whereby the field has a smooth maximum at the centre of the structure and a substantial roll off towards the edges. This is in apparent disagreement with a 3-D EM simulation shown in Figure 7, although the full consequences of this comparison are still under evaluation.

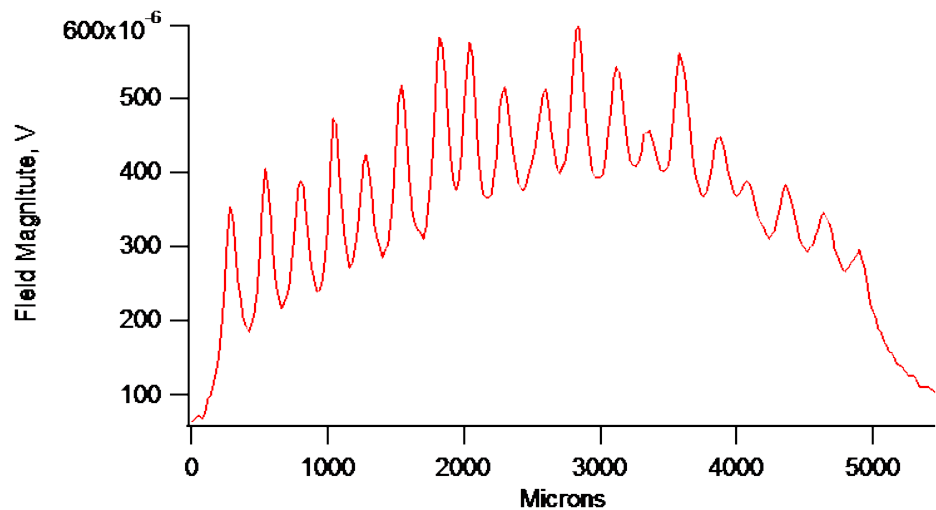

Fig. 5. Voltage distribution transversely along a 19 bond structure.

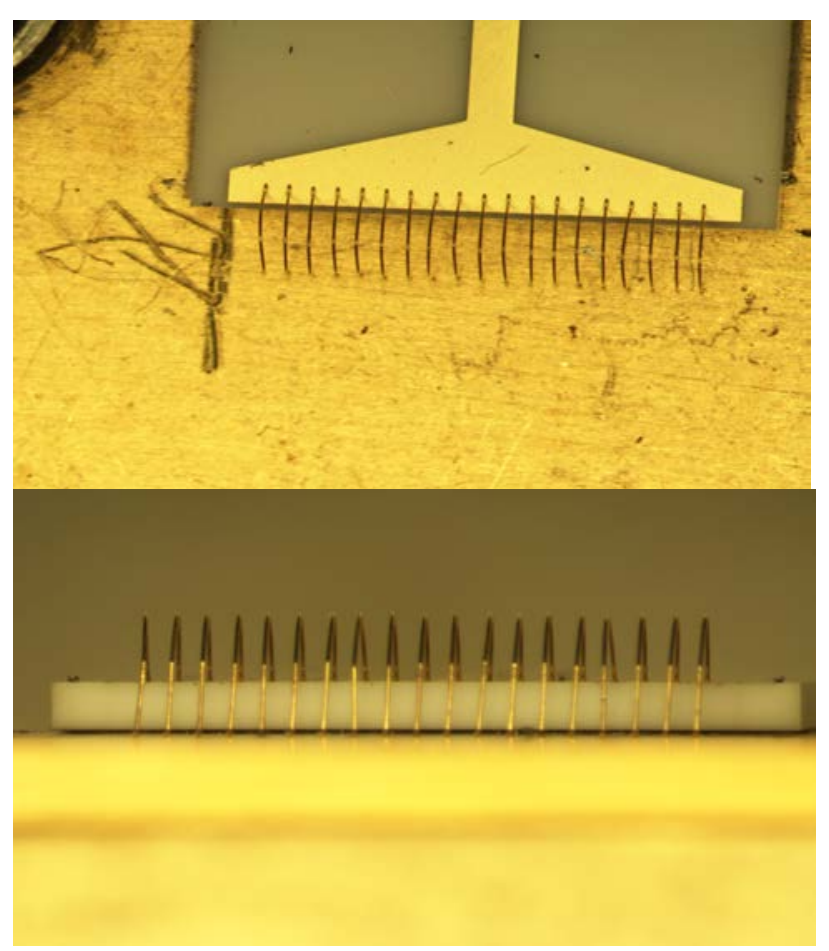

Fig. 6. Plan and side view of a 19 bond test structure.

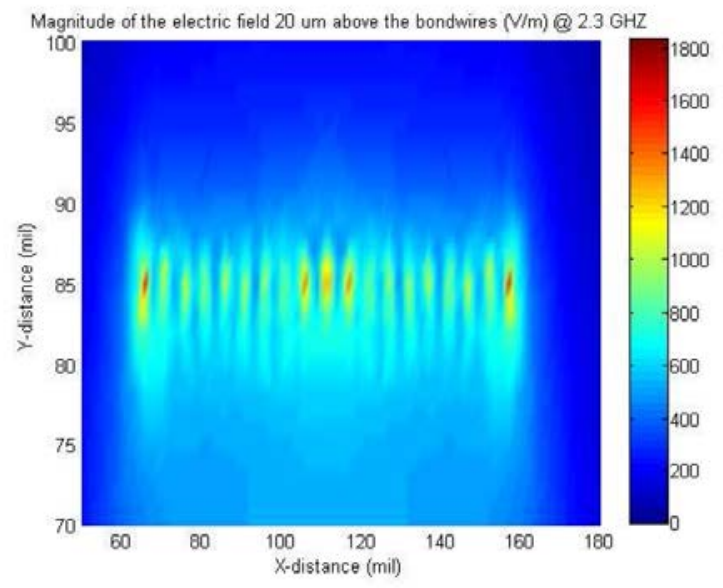

Fig. 7. EM simulation of 19 bond structure.

\section{CONCLUSION}

In conclusion, we have built and demonstrated a novel EField microscope system which has a spatial resolution of better than 100 microns. We believe substantial further improvements can be made to the resolution, for example enabling voltages within RFICs to be probed directly. We are also pursuing applications where the device plane voltages and currents in working PAs (as opposed to devices in load pull systems) can be measured directly. One specific goal in this area is an investigation of device voltages, and potential reliability issues resulting therefrom, in high power Doherty PAs.

\section{REFERENCES}

[1] Cripps S.C, Porch, A., "A High Resolution, Non-Intrusive Field Probe”, Wamicon 2000

[2] Kantor, R., Shvets, I.V., "Measurement of electric field intensities using scanning near-field microwave microscopy”, Trans. Microwave Theory Tech., MTT-51, no. 11, Nov 2003, pp22282234.

[3] H. Barjawi, MSc thesis,2009, Cardiff University, UK.

[4] C. P. Vlahacos, R. C. Black, S. M. Anlage, A. Amar, and F. C. WellstoodAppl. Phys. Lett., Vol. 69, No. 21, 18 November 1996 